\section{Fatores associados ao início tardio da amamentação em hospitais do Sistema Único de Saúde no Município do Rio de Janeiro, Brasil, 2009}

\author{
Factors associated with late initiation of breastfeeding \\ in the Brazilian Unified National Health System in \\ the city of Rio de Janeiro, Brazil, 2009
}

\author{
Factores asociados con el inicio tardío de la \\ lactancia materna en hospitales del Sistema \\ Único de Salud en el municipio de Río de \\ Janeiro, Brasil, 2009
}

Tania Maria Brasil Esteves 1 Regina Paiva Daumas 1 Maria Inês Couto de Oliveira 2 Carlos Augusto Ferreira de Andrade 3 Iuri da Costa Leite 1

\footnotetext{
${ }_{1}^{1}$ Escola Nacional de Saúde Pública Sergio Arouca, Fundação Oswaldo Cruz, Rio de Janeiro, Brasil.

2 Instituto de Saúde da Comunidade, Universidade Federal Fluminense, Niterói, Brasil.

3 Instituto Nacional de Infectologia Evandro Chagas, Fundação Oswaldo Cruz, Rio de Janeiro, Brasil.

Correspondência T. M. B. Esteves Centro de Saúde Escola Germano Sinval Faria, Escola Nacional de Saúde Pública Sergio Arouca, Fundação Oswaldo Cruz. Rua Leopoldo Bulhões 1480, Rio de Janeiro, $R J$ 21041-210, Brasil. tmbesteves@gmail.com
}

\begin{abstract}
This study aimed to analyze factors associated with delayed initiation of breastfeeding (lack of breastfeeding in the first hour of life). This was a cross-sectional study conducted in 2009 with 673 postpartum women at hospitals belonging to the Brazilian Unified National Health System (SUS) in the city of Rio de Janeiro, Brazil. Statistical analysis used multilevel logistic regression, with two levels (individual and hospital). Prevalence of delayed initiation of breastfeeding was 49.2\%. Delivery in a Baby-Friendly Hospital $(B F H)$ had a protective effect against late initiation of breastfeeding $(O R=0.17 ; 95 \% C I$ : $0.05-0.55)$, while cesarean section $(O R=5.95$; 95\%CI: 3.88-9.12) and ignorance of HIV status until delivery $(O R=2.16$; 95\%CI: 1.04-4.50) increased the odds of delay. Reduction in cesarean rates, adherence to protocols to prevent vertical HIV transmission, and expanded accreditation of hospitals in the BFH are important strategies to promote breastfeeding in the first hour of life.
\end{abstract}

Breast Feeding; Postpartum Period; Health Services

\section{Resumo}

O objetivo do trabalho foi analisar os fatores associados com o início tardio da amamentação (não amamentar na primeira hora de vida). Estudo transversal conduzido em 2009 com 673 puérperas internadas em hospitais do Sistema Unico de Saúde (SUS) do Município do Rio de Janeiro, Brasil. Um modelo de regressão logística multinível com dois níveis (individual e hospitalar) foi utilizado nas análises estatísticas. A prevalência de início tardio da amamentação foi de 49,2\%. O parto em Hospital Amigo da Criança (HAC) teve um efeito protetor contra o atraso no início da amamentação $(O R=0,17$; IC95\%: $0,05-0,55)$, enquanto a cesariana $(O R=5,95$; IC95\%: 3,88-9,12) e o desconhecimento do resultado do exame anti-HIV até o parto $(O R=2,16$; IC95\%: 1,04-4,50) aumentaram a chance de atraso. Redução das taxas de cesariana, adesão aos protocolos de atenção pré-natal e ampliação do credenciamento dos hospitais como HAC são estratégias importantes para promover o aleitamento materno na primeira hora de vida.

Aleitamento Materno; Período Pós-Parto; Serviços de Saúde 


\section{Introdução}

O aleitamento materno é considerado uma estratégia-chave para a sobrevivência infantil 1 , reduzindo o risco das principais causas de morbimortalidade em crianças, como a diarreia e a pneumonia 2,3,4. A Iniciativa Hospital Amigo da Criança (IHAC), lançada no início da década de 1990 pelo Fundo das Nações Unidas para a Infância (UNICEF) e a Organização Mundial da Saúde (OMS), tem por objetivo mobilizar os funcionários dos hospitais com leitos obstétricos para o cumprimento dos Dez Passos para o Sucesso do Aleitamento Materno 5.

Entre os "Dez Passos" preconizados pela IHAC para promover a amamentação nos hospitais, o "Passo Quatro" recomenda "Colocar os bebês em contato com a pele da mãe imediatamente após o parto por pelo menos uma hora e incentivar as mães a reconhecer quando seus filhos estão prontos para serem amamentados, oferecendo ajuda se necessário" 6 (p. 153). Essa recomendação baseia-se em estudos que demonstram efeitos fisiológicos positivos do contato pele a pele e do início da amamentação na primeira hora de vida para a mãe 7 e o recém-nascido ${ }^{8}$, maior duração do aleitamento materno 8,9 e redução da mortalidade neonatal 10,11.

No período pós-parto imediato, o recémnascido está mais apto para estabelecer a amamentação 12 , tendo habilidade para rastejar em direção à região mamilo-areolar e sugar espontaneamente nos primeiros sessenta minutos 13 . Após esse período, muitos recém-nascidos entram na fase do sono ${ }^{14}$, prejudicando a amamentação e aumentando o risco da prescrição de complementos 15 .

Em que pesem as recomendações a seu favor, a amamentação na primeira hora de vida ainda não é uma prática incorporada às rotinas hospitalares. Procedimentos que prejudicam o contato precoce mãe/bebê, tais como a aspiração de vias aéreas e a aspiração gástrica, embora desaconselhados para recém-nascidos saudáveis, ainda são realizados rotineiramente por muitos profissionais 16 . O uso de sedativos no período final do parto, a separação mãe/filho e a falta de apoio às mães na sala de parto são outras práticas hospitalares que dificultam o início oportuno da amamentação 17 .

Uma revisão sistemática sobre esse tema apontou a cesariana como o principal fator de risco para a não amamentação na primeira hora de vida 18 . Baixa escolaridade materna, baixa renda familiar, idade materna inferior a 25 anos e ausência de consultas pré-natais foram também reportadas como fatores associados ao início tar- dio da amamentação por pelo menos 2 dos 18 estudos avaliados 18 .

No Brasil, segundo dados da Pesquisa $\mathrm{Na}$ cional de Demografia e Saúde da Criança e da Mulher (PNDS), em 2006, a prevalência de amamentação na primeira hora de vida entre menores de cinco anos era de $43 \%$ no país como um todo e de $37,7 \%$ na Região Sudeste 19 . Outros estudos revelam, porém, que essa prevalência pode apresentar grandes variações entre maternidades localizadas na mesma área geográfica. Estudo realizado em maternidades do Município do Rio de Janeiro, entre 1999 e 2001, identificou uma prevalência de amamentação na primeira hora de vida de apenas $16 \%$, variando de $1,6 \%$ no grupo das maternidades privadas a $39,2 \%$ no grupo das municipais e federais 20. Uma melhor compreensão dos fatores que impedem o início oportuno da amamentação é importante para permitir uma implementação mais efetiva das políticas de promoção do aleitamento materno nas maternidades.

O objetivo do presente trabalho foi analisar os fatores associados ao início tardio da amamentação nas maternidades do Sistema Único de Saúde (SUS) do Município do Rio de Janeiro, considerando o efeito de características individuais (maternas, da atenção pré-natal, da atenção ao parto e do bebê) e do contexto (hospitalares).

\section{Métodos}

Estudo transversal conduzido entre agosto e dezembro de 2009 nos 15 hospitais do SUS do Município do Rio de Janeiro com mais de mil partos/ano, os quais concentravam $94 \%$ do total de partos realizados em unidades hospitalares do SUS em 2008 21. À exceção de um hospital filantrópico, todas as demais unidades eram públicas.

Tendo em vista que a pesquisa previa a análise de diversos desfechos, optou-se por considerar uma prevalência arbitrária de $50 \%$, garantindo assim o maior tamanho de amostra para um dado nível de erro e confiança. Utilizando-se um nível de erro de 5\% e de confiança de $99 \%$, obteve-se um tamanho mínimo de 687 puérperas, distribuídas proporcionalmente entre os 15 hospitais segundo o número de partos/ano 21. Foram consideradas elegíveis para a entrevista as mães com filho nascido vivo há mais de 24 horas, internado em regime de alojamento conjunto, mesmo que o recém-nascido tivesse necessitado anteriormente de cuidados na unidade neonatal. As mães soropositivas para o HIV foram consideradas inelegíveis pela contraindicação ao aleitamento materno vigente em nosso país 22 . 
Em caso de parto gemelar, foram coletadas apenas as informações sobre o recém-nascido mais velho. Para a análise do presente desfecho, o início tardio do aleitamento materno, foram excluídas ainda as mães de bebês com Apgar menor que sete no quinto minuto de vida, pois esta condição poderia impedir a amamentação ao nascimento. A amostra final foi constituída de 673 mães.

As puérperas a serem entrevistadas em cada hospital foram selecionadas, a cada dia, por amostragem sistemática com base na ficha do posto de enfermagem. No caso de recusa, era feita a reposição pela mãe seguinte. As entrevistas ocorreram nos períodos da manhã e da tarde, de segunda-feira a sábado, até que o tamanho amostral calculado para cada hospital fosse atingido. O tempo de coleta de dados em cada hospital variou de duas a seis semanas.

Todas as entrevistas foram realizadas no alojamento conjunto por profissionais de saúde capacitadas previamente na IHAC e treinadas para a aplicação do instrumento de coleta de dados. As atividades de campo foram supervisionadas por enfermeiras capacitadas como avaliadoras da IHAC, credenciadas pelo Ministério da Saúde. As mães foram entrevistadas por meio de questionário adaptado do instrumento para verificação do grau de cumprimento dos "Dez Passos", utilizado nas reavaliações dos Hospitais Amigos da Criança (HAC) 21,23.

A variável desfecho "início tardio da amamentação" foi obtida da pergunta: "Quanto tempo depois do parto seu bebê foi ao peito para mamar pela primeira vez?". Foi considerado início tardio quando o tempo até a primeira mamada foi superior a sessenta minutos.

Tendo em vista que o hospital pode desempenhar um papel importante sobre a chance de a criança ser amamentada na primeira hora de vida e que nem sempre as variáveis hospitalares responsáveis por este efeito são observáveis, a identificação dos fatores associados ao início tardio da amamentação foi feita por meio da utilização de um modelo logístico multinível com dois níveis 24 , sendo o primeiro representado pela mãe e o segundo pela unidade hospitalar. Foram contemplados como variáveis explicativas os fatores associados à amamentação na primeira hora de vida identificados na revisão sistemática 18 que estavam disponíveis na base de dados da pesquisa 21 .

O credenciamento pela IHAC foi a variável analisada no nível hospitalar. No nível individual, foram investigadas as características maternas: idade, escolaridade, cor da pele, viver com companheiro, trabalho remunerado e parturição; as características da atenção pré-natal: realização de pré-natal, trimestre de início do pré-natal, número de consultas; as características da atenção ao parto: conhecimento do resultado do exame anti-HIV antes do parto (qualquer ocasião do pré-natal ou teste rápido na maternidade), tipo de parto, mãe se sentir escutada pela equipe do hospital sobre dúvidas de amamentação; e as características do bebê: sexo e peso ao nascer, que foram expostas em modelo teórico (Figura 1). Foi considerado o resultado do exame realizado em qualquer período do pré-natal pois, na ocasião do estudo, ainda não era vigente a recomendação de realização do teste rápido para HIV na admissão para o parto quando o resultado do 3 o trimestre não está disponível 22 .

Foram calculadas as frequências das covariáveis segundo o tempo até o início da amamentação (na primeira ou após a primeira hora de vida). O teste qui-quadrado de Pearson com a correção por continuidade de Yates foi utilizado para identificar possíveis associações entre as covariáveis e o desfecho "início tardio da amamentação”. As variáveis independentes cujos níveis de significância no teste qui-quadrado mostraram-se menores do que $25 \%$ foram incorporadas à análise multinível, tendo sido interpretadas com base em suas razões de chance (OR) brutas e ajustadas 24 .

A análise dos dados foi realizada utilizandose o software SPSS 17.0 (SPSS Inc., Chicago, Estados Unidos), à exceção da análise multinível, implementada no software MLwiN 2.26 (Centre for Multilevel Modelling, University of Bristol, Bristol, Reino Unido). Os parâmetros dos modelos foram estimados usando-se o método de quaseverossimilhança penalizada de segunda ordem, considerado como o melhor procedimento de estimação para modelos multiníveis com resposta binária, disponível no MLwiN 25.

\section{Resultados}

Foram incluídas no estudo 673 puérperas entrevistadas em 15 hospitais, dos quais sete eram credenciados pela IHAC. Houve sete recusas. As características das mães, dos recém-nascidos e da assistência pré-natal e hospitalar são apresentadas na Tabela 1, assim como a prevalência de início tardio da amamentação segundo estas características. No total da amostra, a prevalência de início tardio da amamentação foi de $49,2 \%$. Pouco mais da metade (51\%) das entrevistas ocorreram em hospitais credenciados pela IHAC. Uma em cada quatro mães era adolescente e $31,9 \%$ não completaram o ensino fundamental. Quase a totalidade das mães recebeu acompanhamento pré-natal, embora 22,9\% tenham 


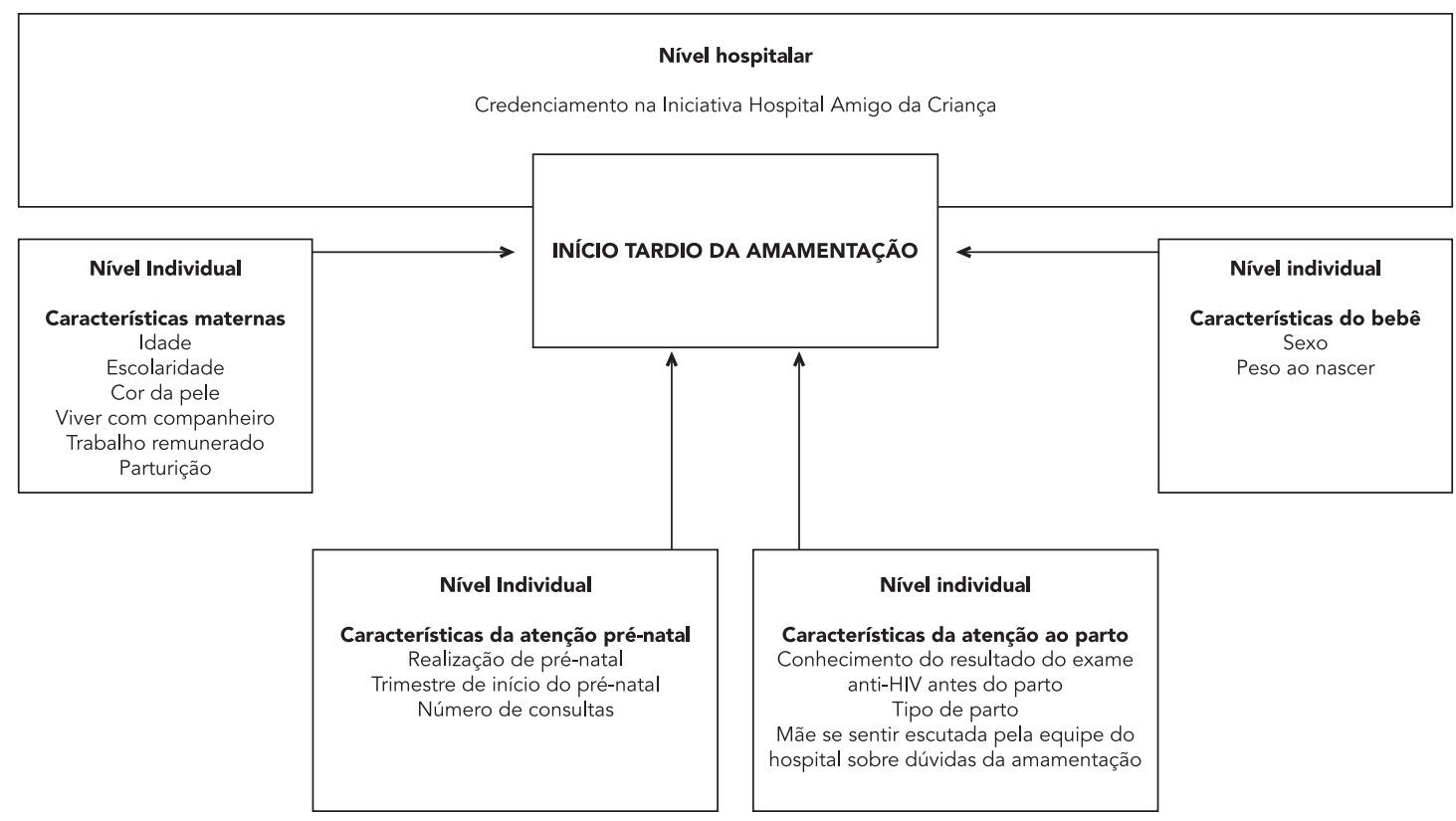

sido consultadas menos de seis vezes durante a gestação. Cerca de um terço das mães foi submetida à cesariana. A maioria $(87,8 \%)$ declarou ter realizado exame anti-HIV no pré-natal e $89,5 \%$ sabiam do resultado de algum teste anti-HIV antes do parto (exame pré-natal ou teste rápido na maternidade).

O início tardio da amamentação foi significativamente maior entre mães que não realizaram o pré-natal $(79,2 \%)$, submetidas à cesariana $(70 \%)$, que desconheciam o seu status sorológico para o HIV na hora do parto $(66,2 \%)$ ou que declararam que a equipe do hospital não havia escutado suas dúvidas sobre amamentação $(59,2 \%)$. Nos hospitais credenciados na IHAC, o início tardio foi menos frequente $(32,7 \%)$ do que nos demais hospitais $(66,4 \%)$. Idade materna e escolaridade estiveram também associadas ao início tardio da amamentação no nível de significância de 25\%, sendo selecionadas para inclusão nos modelos de regressão multinível.

A Tabela 2 apresenta as razões de chance brutas e ajustadas para cada uma das variáveis previamente selecionadas, obtidas pelos modelos de regressão logística multinível. No modelo de regressão simples, ajustado apenas pelo efeito aleatório (hospitais), idade materna adolescente e parto em HAC foram fatores de proteção contra o início tardio da amamentação. Por outro lado, não ter realizado acompanhamento pré-natal, parir por cesariana e desconhecer o status sorológico para o HIV no momento do parto aumentaram as chances do desfecho.

No modelo de regressão múltipla, entre as variáveis medidas no nível individual, a cesariana $(\mathrm{OR}=5,95$; IC95\%: 3,88-9,12) e o desconhecimento do resultado da testagem anti-HIV antes do parto (OR = 2,16; IC95\%:1,04-4,50) foram os fatores que aumentaram significativamente a chance de início tardio da amamentação. No nível hospitalar, a certificação do hospital pela IHAC foi um importante fator de proteção contra o início tardio da amamentação, reduzindo em $83 \%$ a chance deste desfecho $(\mathrm{OR}=0,17$; IC95\%: 0,05-0,55). Mesmo após a inclusão de variáveis individuais e do hospital, o desvio padrão do efeito aleatório permaneceu estatisticamente significativo (OR = 2,95; IC95\%: 1,56-4,33), indicando que a chance de não amamentar entre as mães que tiveram seus filhos em hospitais situados a um desvio padrão acima da média é quase três vezes a de mães que tiveram seus filhos em hospitais situados na média da distribuição. Na Figura 2, são apresentados os efeitos aleatórios 
Tabela 1

Distribuição de frequência das características sociodemográficas, do pré-natal, do parto e do bebê, e prevalência de início tardio da amamentação segundo estas características. Município do Rio de Janeiro, Brasil, 2009.

\begin{tabular}{|c|c|c|c|c|c|}
\hline & \multicolumn{2}{|c|}{ Frequência } & \multicolumn{2}{|c|}{ Prevalência de início tardio } & \multirow[t]{2}{*}{ Valor de $p$ * } \\
\hline & $\mathrm{n}$ & $\%$ & n & $\%$ & \\
\hline \multicolumn{6}{|l|}{ Idade da mãe (anos) } \\
\hline $13-19$ & 174 & 25,9 & 75 & 43,1 & 0,06 \\
\hline 20 e mais & 499 & 74,1 & 256 & 51,3 & \\
\hline \multicolumn{6}{|c|}{ Escolaridade (anos de estudos) } \\
\hline $0-7$ & 215 & 31,9 & 114 & 63,0 & 0,200 \\
\hline 8 ou mais & 458 & 68,1 & 217 & 47,4 & \\
\hline \multicolumn{6}{|l|}{ Cor da pele da mãe } \\
\hline Branca & 168 & 25,0 & 81 & 48,2 & 0,841 \\
\hline Não branca & 505 & 75,0 & 250 & 49,5 & \\
\hline \multicolumn{6}{|l|}{ Vive com companheiro } \\
\hline $\operatorname{Sim}$ & 504 & 74,9 & 247 & 49,0 & 0,946 \\
\hline Não & 169 & 25,1 & 84 & 49,7 & \\
\hline \multicolumn{6}{|c|}{ Mãe tem trabalho remunerado } \\
\hline Sim & 334 & 49,6 & 165 & 49,4 & 0,972 \\
\hline Não & 339 & 50,4 & 166 & 49,0 & \\
\hline \multicolumn{6}{|l|}{ Parturição } \\
\hline Multíparas & 351 & 52,2 & 168 & 47,9 & 0,524 \\
\hline Primíparas & 322 & 47,8 & 163 & 50,6 & \\
\hline \multicolumn{6}{|l|}{ Realizou pré-natal } \\
\hline Sim & 649 & 96,4 & 312 & 48,1 & 0,005 \\
\hline Não & 24 & 3,6 & 19 & 79,2 & \\
\hline \multicolumn{6}{|c|}{ Início do pré-natal no primeiro trimestre } \\
\hline $\operatorname{Sim}$ & 453 & 67,3 & 320 & 48,6 & 0,706 \\
\hline Não & 220 & 32,7 & 111 & 50,5 & \\
\hline \multicolumn{6}{|c|}{ 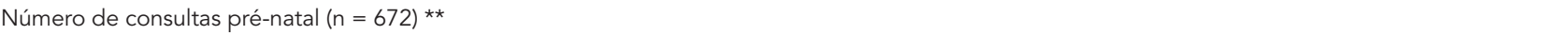 } \\
\hline $0-5$ & 154 & 22,9 & 80 & 51,9 & 0,464 \\
\hline 6 ou mais & 518 & 77,1 & 251 & 48,5 & \\
\hline \multicolumn{6}{|l|}{ Tipo de parto } \\
\hline Vaginal & 456 & 67,8 & 179 & 39,3 & $<0,001$ \\
\hline Cesariana & 217 & 37,2 & 152 & 70,0 & \\
\hline \multirow{2}{*}{\multicolumn{6}{|c|}{$\begin{array}{l}\text { Na hora do parto, a mãe sabia seu status sorológico para o HIV (pré-natal ou } \\
\text { teste rápido)? }\end{array}$}} \\
\hline & & & & & \\
\hline Sim (HIV negativo) & 602 & 89,5 & 284 & 47,2 & 0,004 \\
\hline Não (ignorado) & 71 & 10,5 & 47 & 66,2 & \\
\hline \multicolumn{6}{|c|}{ Equipe escuta as dúvidas sobre amamentação? $(n=672)$ ** } \\
\hline Sim & 503 & 74,9 & 230 & 45,7 & 0,003 \\
\hline Não/Mais ou menos & 169 & 25,1 & 100 & 59,2 & \\
\hline \multicolumn{6}{|l|}{ Sexo do bebê } \\
\hline Feminino & 323 & 48,0 & 156 & 48,3 & 0,716 \\
\hline Masculino & 350 & 52,0 & 175 & 50,0 & \\
\hline \multicolumn{6}{|c|}{ Baixo peso ao nascer $(n=672) * \star$} \\
\hline Sim & 45 & 6,7 & 24 & 53,3 & 0,665 \\
\hline Não & 627 & 93,3 & 306 & 48,8 & \\
\hline \multicolumn{6}{|c|}{ Hospital Amigo da Criança } \\
\hline Sim & 343 & 51,0 & 112 & 32,7 & $<0,001$ \\
\hline Não & 330 & 49,0 & 219 & 66,4 & \\
\hline Total & 673 & 100,0 & 331 & 49,2 & \\
\hline
\end{tabular}

* Valor de p da comparação das categorias de cada variável calculado pelo teste de qui-quadrado;

** Variáveis em que houve missing. 
Distribuição das razões de chances (OR) brutas e ajustadas para o início tardio da amamentação de vida nos modelos de regressão logística multinível. Município do Rio de Janeiro, Brasil, 2009 ( $n=673$ ).

\begin{tabular}{|c|c|c|c|c|}
\hline & \multicolumn{2}{|c|}{ Regressão simples * } & \multicolumn{2}{|c|}{ Regressão múltipla } \\
\hline & OR bruta & IC95\% & OR ajustada & IC95\% \\
\hline \multicolumn{5}{|l|}{ Características individuais } \\
\hline \multicolumn{5}{|l|}{ Idade materna (anos) } \\
\hline $13-19$ & 0,61 & $0,41-0,92$ & 0,65 & $0,41-1,03$ \\
\hline 20 e mais & 1,00 & & 1,00 & \\
\hline \multicolumn{5}{|c|}{ Escolaridade materna (anos de estudos) } \\
\hline $0-7$ & 1,38 & $0,95-2,01$ & 1,43 & $0,95-2,17$ \\
\hline 8 ou mais & 1,00 & & 1,00 & \\
\hline \multicolumn{5}{|l|}{ Realizou pré-natal } \\
\hline Não & 3,32 & $1,05-10,52$ & 2,36 & $0,64-8,61$ \\
\hline Sim & 1,00 & & 1,00 & \\
\hline \multicolumn{5}{|l|}{ Cesariana } \\
\hline Sim & 5,72 & $3,77-8,68$ & 5,95 & $3,88-9,12$ \\
\hline Não & 1,00 & & 1,00 & \\
\hline \multicolumn{5}{|c|}{ Na hora do parto, a mãe sabia seu status sorológico } \\
\hline \multicolumn{5}{|c|}{ para o HIV? } \\
\hline Não & 2,21 & $1,20-4,09$ & 2,16 & $1,04-4,50$ \\
\hline $\operatorname{Sim}$ & 1,00 & & 1,00 & \\
\hline \multicolumn{5}{|l|}{ Escutam dúvidas? } \\
\hline Não ou mais ou menos & 1,39 & $0,91-2,11$ & 1,43 & $0,90-2,26$ \\
\hline Sim & 1,00 & & 1,00 & \\
\hline \multicolumn{5}{|c|}{ Características do hospital (contexto) } \\
\hline \multicolumn{5}{|l|}{ Hospital Amigo da Criança } \\
\hline $\operatorname{Sim}$ & 0,19 & $0,06-0,54$ & 0,17 & $0,05-0,55$ \\
\hline Não & 1,00 & & 1,00 & \\
\hline Efeito aleatório & 3,66 & $1,82-5,66$ & 2,95 & $1,56-4,33$ \\
\hline
\end{tabular}

IC95\%: intervalo de 95\% de confiança.

* Medidas de associação ajustadas apenas pelo efeito aleatório do nível hospitalar

referentes a cada um dos hospitais considerados neste estudo, em ordem crescente segundo seus impactos sobre a chance de início tardio da amamentação. Conforme pode ser visto, há quatro hospitais com efeitos aleatórios significativamente diferentes de zero: um com efeito de proteção e três de risco.

\section{Discussão}

Este trabalho, elaborado para analisar os fatores associados ao início tardio da amamentação nas maternidades do SUS do Município do Rio de Janeiro, mostrou que pouco mais da metade das mulheres que deram à luz no segundo semestre de 2009 iniciaram a amamentação na primeira hora de vida. Essa proporção foi significativamente mais elevada nos hospitais credenciados pela IHAC (67,3\%), demostrando a importância desta iniciativa. $\mathrm{O}$ nascimento em hospital não credenciado na IHAC, o parto por cesariana e o desconhecimento do status sorológico para o HIV foram as condições identificadas como fatores de risco independentes para o início tardio da amamentação.

A prevalência da amamentação na primeira hora de vida nesta pesquisa $(50,8 \%)$ foi um pouco superior à nacional, observada em 2006 (43\%). Foi também superior à prevalência encontrada em maternidades federais e municipais do $\mathrm{Mu}$ nicípio do Rio de Janeiro, em estudo realizado de 1999 a $2001(39,2 \%)$ 20. Esse resultado pode ser devido a diferenças nas maternidades incluídas nos estudos, podendo refletir também os avanços da IHAC no Município do Rio de Janeiro no período 26 . 
Efeitos aleatórios referentes a cada um dos hospitais, em ordem crescente segundo seus impactos sobre a chance de início tardio da amamentação.

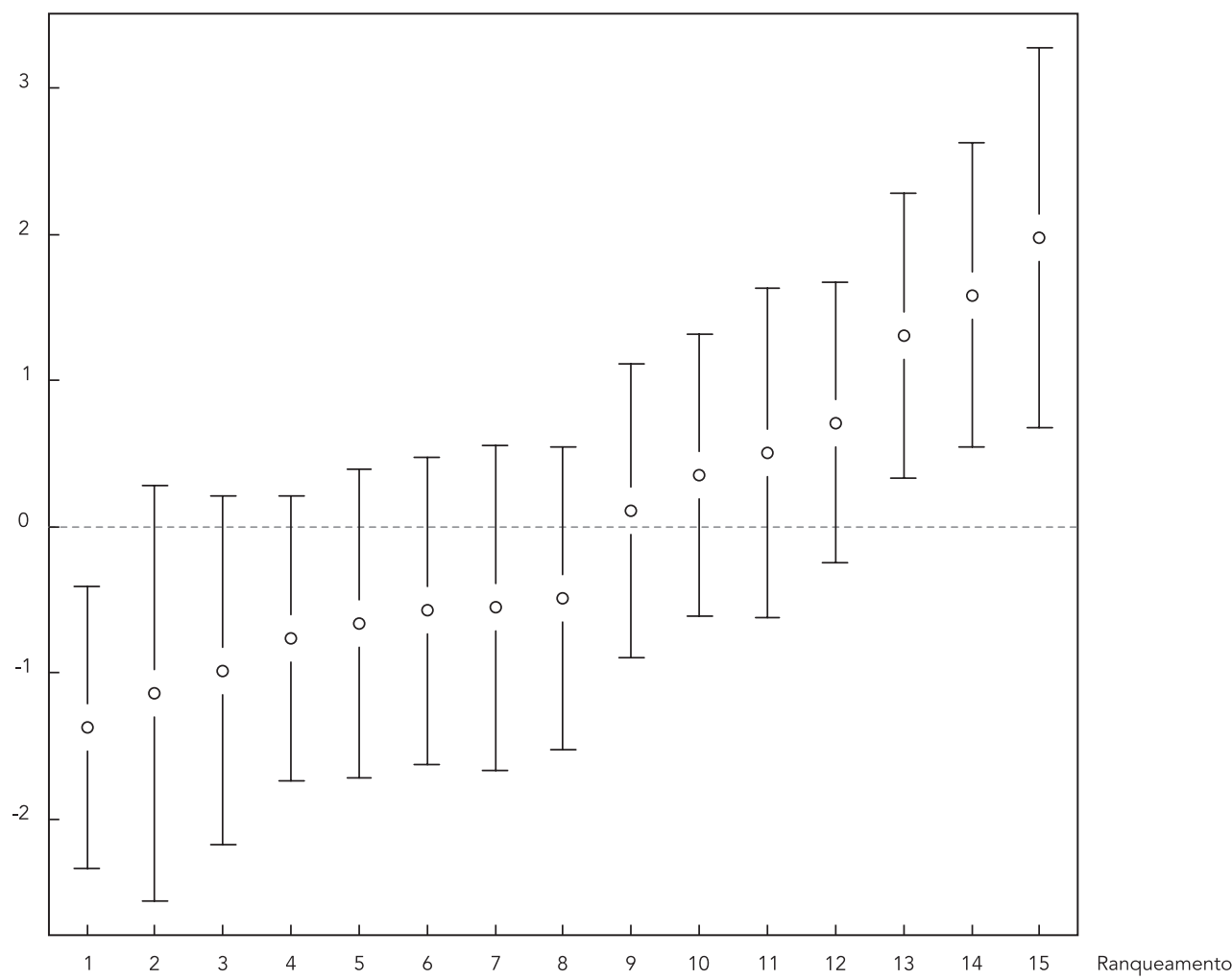

No presente trabalho, a cesariana foi o fator de risco mais fortemente associado ao início tardio da amamentação, corroborando o resultado de revisão sistemática sobre este tema, na qual este foi o fator de risco identificado de forma mais consistente ${ }^{18}$. Essa modalidade de parto tem sido descrita como uma importante barreira para o início oportuno da amamentação 27 , em geral devido às rotinas de cuidados pós-operatórios que retardam ou interrompem o contato entre mãe e filho no período pós-parto ${ }^{28}$. A proporção de cesarianas em nosso estudo $(32,2 \%)$ foi mais que o dobro da considerada aceitável pela OMS (10 a 15\%) 29, embora inferior à encontrada no mesmo município por Boccolini et al. 20 (49,4\%) em estudo que incluiu a rede hospitalar privada. Embora a cesariana aumente o risco de morte materna, infecções puerperais e prematuridade, a proporção de partos por esta via tem aumentado, sendo motivo de preocupação em todo o mundo 30 . No Brasil, no ano de 2010, 52\% dos partos ocorreram por cesariana, a despeito das iniciativas governamentais para reduzir a ocorrência deste tipo de parto 31 .

O desconhecimento do status sorológico para o HIV foi outra variável do nível individual identificada como fator de risco independente para o início tardio da amamentação. A testagem para o HIV no pré-natal e na maternidade é essencial para a prevenção da transmissão vertical do vírus 32. Apesar de o nosso país contar com um programa com protocolos bem estabelecidos de prevenção e controle do HIV/AIDS 33, falhas nos processos envolvidos ainda são encontradas 22. Considerando a disponibilidade do teste rápido anti-HIV nas maternidades investigadas, com possibilidade de resultados em menos de trinta minutos 22 , o fato de cerca de $10 \%$ das parturientes chegarem ao momento do parto desconhecendo sua sorologia sugere a necessidade de melhoria nos processos de comunicação entre laboratório, equipe de saúde e pacientes, já apontada por pesquisa anterior 34 . 
Deve-se ressaltar que o desconhecimento do status sorológico para o HIV pela mãe, na hora do parto, poderia ser considerado também como uma condição marcadora de acompanhamento pré-natal inadequado ou de baixo conhecimento da mãe sobre questões de saúde, visto que as mães nesta condição desconheciam qualquer resultado de exame para HIV do pré-natal, além de não saberem do resultado do teste rápido da maternidade. Quase a metade dessas mães não havia mesmo realizado qualquer consulta pré-natal.

O credenciamento do hospital pela IHAC foi o fator de proteção mais importante contra o início tardio da amamentação em nosso estudo, corroborando resultados de pesquisas anteriores 35,36. A IHAC foi criada para encorajar o estabelecimento dos "Dez Passos" mundialmente, oferecendo materiais que incluem orientações para o planejamento de programas nacionais, treinamento para equipes clínicas e gestores hospitalares, formulários de autoavaliação e, finalmente, o credenciamento dos hospitais como "Amigos da Criança" (HAC) 37. Após o credenciamento, ações de educação permanente e monitoramento contínuo são essenciais ${ }^{38}$, visto que o grau de cumprimento das ações preconizadas tende a decair ao longo do tempo ${ }^{21}$, podendo reduzir o impacto da estratégia 36 .

Apesar de o Brasil ter instituído um programa nacional de aleitamento materno desde 1981 e ter adotado a IHAC como política desde 1992, ainda não foi alcançada a meta de credenciar como HAC pelo menos $50 \%$ dos hospitais com mais de mil partos/ano 26. Diante da demonstrada efetividade das ações da IHAC 39, é importante identificar e ultrapassar os entraves à adoção desta política pelos hospitais 26 .

Nas análises realizadas, foi identificado um efeito de contexto (dos hospitais) significativo sobre o início tardio da amamentação, mostrando que a unidade de saúde onde ocorre o parto tem um papel importante na determinação deste desfecho. Esse efeito não foi totalmente explicado nem pelo credenciamento na IHAC, nem pelas outras variáveis do modelo, sugerindo que outras características dos hospitais estariam determinando as diferenças entre eles quanto à chance de início tardio da amamentação.

Algumas pesquisas identificaram a ausência de assistência pré-natal como um fator de risco para o início tardio da amamentação ${ }^{18}$. No presente estudo, esse fator só esteve associado ao desfecho nas análises não ajustadas, provavelmente devido à sua associação com o conhecimento do status sorológico para o HIV.

A idade materna não foi um fator de risco independente para o início tardio da amamen- tação na população do estudo. Contudo, na regressão simples, as mães adolescentes tiveram uma maior chance de início oportuno da amamentação. É provável que a menor proporção de cesarianas nesse grupo $(25,3 \%$ vs. $34,7 \%$; $\mathrm{p}=$ 0,024 , dados não apresentados) tenha contribuído para esse efeito.

Destaca-se, como ponto forte deste trabalho, a utilização de uma amostra representativa das parturientes internadas nas maternidades que concentram $94 \%$ dos partos realizados pelo SUS no Município do Rio de Janeiro, traçando um panorama global da prática do aleitamento materno na primeira hora nesta rede hospitalar. Além disso, a análise dos dados com um modelo de regressão multinível permitiu estimar os parâmetros associados com as variáveis dos níveis individual e hospitalar, bem como o próprio efeito de contexto (hospital), considerando a estrutura hierárquica entre eles.

Algumas limitações do presente estudo devem ser consideradas. $\mathrm{O}$ conhecimento do resultado do teste rápido anti-HIV por parte da equipe assistente não foi aferido, não sendo possível determinar em que medida o desconhecimento do seu resultado pela mãe reflete problemas no processo de testagem na maternidade ou falhas no aconselhamento pós-teste. Há também imprecisão e potencial erro de classificação na informação sobre o tempo decorrido até a primeira mamada, uma vez que este dado foi estimado pelas mães. Contudo, esse problema foi minimizado pela realização das entrevistas na maternidade, geralmente nas primeiras 48 horas após o parto.

Os resultados deste trabalho sugerem que os fatores relacionados à assistência pré-natal e ao parto são aqueles que exercem maior influência sobre o início oportuno da amamentação. Embora a continuidade do aleitamento materno seja dependente de uma complexa rede de determinantes sociais e culturais, nos partos hospitalares o início da amamentação ao nascimento seria fortemente dependente das práticas instituídas pela maternidade 20 . Considerando a vulnerabilidade das mães no período pós-parto imediato, cabe à unidade de saúde a responsabilidade de promover a amamentação oportuna e as boas práticas de cuidado à mulher e ao recém-nascido 40 .

Entre as ações potencialmente efetivas para promover o início da amamentação na primeira hora de vida, identificamos: (1) a ampliação do credenciamento dos hospitais na IHAC, com ações de treinamento e monitoramento periódicos que garantam a manutenção dos passos cumpridos; (2) a redução das cesarianas eletivas; e (3) a operacionalização mais efetiva dos procedimentos preconizados para o diagnóstico sorológico do HIV na assistência pré-natal e ao parto. 
Além de serem importantes para a promoção do aleitamento materno, essas são ações de qualificação da assistência pré-natal e ao parto na perspectiva da integralidade e da humanização da atenção. Por seu potencial de promover a saúde e reduzir a morbimortalidade materna e infantil, devem ser consideradas prioridades na definição das políticas públicas voltadas para a saúde da mulher e da criança.

\section{Resumen}

El objetivo del estudio fue analizar los factores asociados al inicio tardio de la lactancia materna (no dar el pecho durante la primera hora de vida). Se trata de un estudio transversal que se llevó a cabo en el 2009 con 673 mujeres que dieron a luz internadas en hospitales del Sistema Único de Salud (SUS) del Municipio de Río de Janeiro, Brasil. Se utilizó un modelo de regresión logística multinivel con dos niveles (individual y hospitalario) en los análisis estadísticos. La prevalencia del inicio tardio de la lactancia fue del 49,2\%. El parto en un Hospital Amigo del Niño (HAN) tuvo un efecto protector contra el inicio tardio de la lactancia $(O R=0,17 ;$ IC95\%: 0,05-0,55), mientras que la cesárea $(O R=5,95$; IC95\%: 3,88-9,12) y el desconocimiento del resultado del examen anti-VIH hasta el parto $(O R=$ 2,16; IC95\%: 1,04-4,50) aumentaron la oportunidad de su aplazamiento. La reducción de las tasas de cesárea, la adhesión a los protocolos de atención prenatal y la ampliación de la acreditación de los hospitales del tipo HAN son importantes estrategias para promover la lactancia materna en la primera hora de vida.

Lactancia Materna; Periodo Posparto; Servicios de Salud

\section{Colaboradores}

T. M. B. Esteves participou da aquisição, análise e interpretação dos dados e da redação do manuscrito. $\mathrm{R}$. P. Daumas participou da análise e interpretação dos dados, redação e revisão do manuscrito. M. I. C. Oliveira participou da concepção do estudo, análise e interpretação dos dados, redação e revisão do manuscrito. C. A. F. Andrade e I. C. Leite participaram da análise e interpretação dos dados e revisão crítica do manuscrito.

\section{Agradecimentos}

Os autores agradecem ao CNPq pelo apoio financeiro fornecido por meio do Edital no 57/2008 à pesquisa coordenada pela Universidade Federal Fluminense, que deu origem a este artigo. 


\section{Referências}

1. United Nations Children's Fund. UNICEF and the Global Strategy on Infant and Young Child Feeding (GSIYCF): understanding the past - planning the future. (UNICEF Working Paper). http://www. unicef.org/nutrition/files/FinalReportonDistribu tion.pdf (acessado em Ago/2013).

2. Cesar JA, Victora CG, Barros FC, Santos IS, Flores JA. Impact of breast feeding on admission for pneumonia during postneonatal period in Brazil: nested case-control study. BMJ 1999; 318:1316-20.

3. Victora CG, Smith PG, Vaughan JP, Nobre LC, Lombardi C, Teixeira AMB, et al. Evidence for protection by breastfeeding against infants deaths from infectious diseases in Brazil. Lancet 1987; 2: 319-22.

4. Lamberti LM, Walker CLF, Noiman A, Victora CG, Black RE. Breastfeeding and the risk for diarrhea morbidity and mortality. BMC Public Health 2011; 11 Suppl 3:S15.

5. World Health Organization; United Nations Children's Fund. The global criteria for the WHO/UNICEF Baby-Friendly Hospital Initiative. Geneva: World Health Organization; 1992.

6. Fundo das Nações Unidas para a Infância. Iniciativa Hospital Amigo da Criança: revista, atualizada e ampliada para o cuidado integrado. Módulo 2: fortalecendo e sustentando a Iniciativa Hospital Amigo da Criança: um curso para gestores. Brasília: Ministério da Saúde; 2009. (Série A. Normas e Manuais Técnicos).

7. Widström AM, WahlbergV, Matthiesen AS, Eneroth P, Uvnäs-Moberg K, Werner S, et al. Short-term effects of early suckling and touch of the nipple on maternal behaviour. Early Hum Dev 1990; 21:15363.

8. Moore ER, Anderson GC, Bergman N, Dowswell T. Early skin-to-skin contact for mothers and their healthy newborn infants. Cochrane Database Syst Rev 2012; (5):CD003519.

9. Taylor PM, Maloni JA, Brown DR. Early suckling and prolonged breast-feeding. Am J Dis Child 1986; 140:151-4

10. Edmond KM, Zandoh C, Quigley MA, AmengaEtego S, Owusu-Agyei S, Kirkwood BR. Delayed breastfeeding initiation increases risk of neonatal mortality. Pediatrics 2006; 117:e380-6.

11. Boccolini CS, Carvalho ML, Oliveira MIC, PérezEscamilla R. Breastfeeding during the first hour of life and neonatal mortality. J Pediatr 2013; 89: 131-6.

12. Akré J. Infant feeding. The physiological basis. Bull World Health Organ 1994; 67 Suppl:1-108.

13. Righard L, Alade MO. Effect of delivery room routines on success of first breastfeed. Lancet 1990; 336:1105-7.

14. Widström AM, Lilja G, Aaltomaa-Michalias P, Dahllöf A, Lintula M, Nissen E. Newborn behaviour to locate the breast when skin-to-skin: a possible method forenabling early self-regulation. Acta Paediatr 2011; 100:79-85.
15. Lopes FO, Oliveira MIC, Brito AS, Fonseca VM. Fatores associados ao uso de suplementos em recém-natos em alojamento conjunto no Município do Rio de Janeiro. Ciênc Saúde Coletiva 2013; 18:431-9.

16. Mercer JS, Erickson-Owens DA, Graves B, Haley MM. Evidence-based practices for the fetal to newborn transition. J Midwifery Womens Health 2007; 52:262-72.

17. Forster DA, McLachlan HL. Breastfeeding initiation and birth setting practices: a review of the literature. J Midwifery Womens Health 2007; 52: 273-80.

18. Esteves TMB, Daumas RP, Oliveira MIC, Andrade CAF, Leite IC. Determinantes da amamentação na primeira hora de vida: uma revisão sistemática. Rev Saúde Pública 2014; 48:697-708.

19. Ministério da Saúde. Pesquisa Nacional de Demografia e Saúde da Criança e da Mulher - PNDS 2006: dimensões do processo reprodutivo e da saúde da criança. Brasília: Ministério da Saúde; 2009. (Série G, Estatística e Informação em Saúde).

20. Boccolini CS, Carvalho ML, Oliveira MIC, Vasconcellos AGG. Fatores associados à amamentação na primeira hora de vida. Rev Saúde Pública 2011; 45:69-78.

21. Oliveira MIC, Hartz ZMA, Nascimento VC, Silva KS. Avaliação da implantação da iniciativa hospital amigo da criança no Rio de Janeiro, Brasil. Rev Bras Saúde Matern Infant 2012; 12:281-95.

22. Programa Nacional de DST e Aids, Secretaria de Vigilância em Saúde, Ministério da Saúde. Recomendações para profilaxia da transmissão vertical do HIV e terapia antirretroviral em gestantes: manual de bolso. Brasília: Ministério da Saúde; 2010.

23. Fundo das Nações Unidas para a Infância. Iniciativa Hospital Amigo da Criança: revista, atualizada e ampliada para o cuidado integrado. Módulo 5: avaliação e reavaliação externa. Brasília: Ministério da Saúde; 2010.

24. Krogsta U, Hofoss D, Veenstra M, Gulbrandsen P, Hjortdahl P. Hospital quality improvement in context: a multilevel analysis of staff job evaluations. Qual Saf Health Care 2005; 14:438-42.

25. Goldstein JJ, Rasbash J. Improved approximations for multilevel models with binary response. J R Stat Soc Ser A Stat Soc 1996; 159:505-13.

26. Rea MF. Reflexões sobre a amamentação no Brasil: de como passamos a 10 meses de duração. Cad Saúde Pública 2003; 19 Suppl 1:S37-45.

27. Rowe-Murray HJ, Fisher JR. Baby friendly hospital practices: cesarean section is a persistent barrier to early initiation of breastfeeding. Birth 2002; 29:124-31.

28. Prior E, Santhakumaran S, Gale C, Philipps LH, Modi N, Hyde MJ. Breastfeeding after cesarean delivery: a systematic review and meta-analysis of world literature. Am J Clin Nutr 2012; 95:1113-35. 
29. Appropriate technology for birth. Lancet 1985; 2:436-7.

30. Gibbons L, Belizán JM, Lauer JA, Betrán AP, Merialdi $\mathrm{M}$, Althabe $\mathrm{F}$. The global numbers and costs of additionally needed and unnecessary caesarean sections performed per year: overuse as a barrier to universal coverage. Geneva: World Health Organization; 2010. (Background Paper, 30).

31. Ministério da Saúde. As cesarianas no Brasil: situação no ano de 2010, tendências e perspectivas. Saúde Brasil 2010: uma análise da situação de saúde e de evidências selecionadas de impacto de ações de vigilância em saúde. Brasília: Ministério da Saúde; 2011.

32. Dunn TDT, Newll ML, Ades AE, Peckham CS. Risk of human immunodeficiency virus type 1 transmission through breastfeeding. Lancet 1992; 340:585-8.

33. Szwarcwald CL, Castilho EA. The HIV/AIDS epidemic in Brazil: three decades. Cad Saúde Pública 2011; 27 Suppl 1:S4-5.

34. Oliveira MIC, Silva KS, Gomes-Jr. SC, Fonseca VM. Resultado do teste rápido anti-HIV após o parto: uma ameaça à amamentação ao nascimento. Rev Saúde Pública 2010; 44:60-9.

35. Silveira RB, Albernaz E, Zuccheto LM. Fatores associados ao início da amamentação em uma cidade do sul do Brasil. Rev Bras Saúde Matern Infant 2008; 8:35-43.
36. Hawkins SS, Stern AD, Baum CF, Gillman MW. Compliance with the Baby-Friendly Hospital Initiative and impact on breastfeeding rates. Arch Dis Child Fetal Neonatal Ed 2014; 99:F138-43.

37. Labbok MH. Global Baby-Friendly Hospital Initiative monitoring data: update and discussion. Breastfeed Med 2012; 7:210-22.

38. Schmied V, Gribble K, Sheehan A, Taylor C, Dykes FC. Ten steps or climbing a mountain: a study of Australian health professionals' perceptions of implementing the baby friendly health initiative to protect, promote and support breastfeeding. BMC Health Serv Res 2011; 11:208-32.

39. Declercq E, Labbok MH, Sakala C, O'Hara M. Hospital practices and women's likelihood of fulfilling their intention to exclusively breastfeed. Am J Public Health 2009; 99:929-35.

40. Ministério da Saúde. Portaria MS/GM no 1.153, de 22 de maio de 2014. Redefine os critérios de habilitação da Iniciativa Hospital Amigo da Criança (IHAC) como estratégia de promoção, proteção e apoio ao aleitamento materno e à saúde integral da criança e da mulher, no âmbito do Sistema Único de Saúde (SUS). Diário Oficial da União 2014; 28 mai.

Recebido em 14/Ago/2014

Versão final reapresentada em 17/Abr/2015

Aprovado em 04/Mai/2015 